\title{
The evolution of antibiotic resistance
}

\section{Clinically relevant evolution studies are needed to help fight the spread of antibiotic re-} sistance

By R. Craig MacLean ${ }^{1, *}$ and Alvaro San Millan²

For most of human history, bacterial pathogens have been a major cause of disease and mortality. The development of antibiotics provided a simple and effective treatment for bacterial infections, and they have since had huge effects on human health and longevity. This is threatened with the rise of antibiotic resistance (AMR): Many pathogenic bacteria have evolved resistance to the main classes of antibiotics and pan-resistant bacteria have caused untreatable infections. AMR already imposes substantial health and economic problems, and the global annual burden of AMR could increase to 10 million deaths and 100 Trillion USD by 2050 (1). Understanding how AMR evolves and spreads is therefore key to improving antibiotic treatment strategies.

Two main approaches have been used to understand the processes underlying the spread of AMR. Clinical studies have focused on DNA sequencing to identify the genes underlying AMR, and the epidemiological dynamics of resistance. Experimental studies have focused on simple in vitro model systems to investigate how AMR changes in response to defined and controlled selective pressures, often supported by mathematical models (3). However, it has become increasingly clear that there are substantial gaps between these two approaches. Put simply, experimental studies have not placed enough emphasis on understanding the evolution of successful strains that have driven the rise of resistance in the clinic. To bridge these gaps and identify strategies to counteract AMR, experimental studies should shift focus towards investigating clinically-relevant resistance genes, and work with more realistic infection models.

Pathogenic bacteria acquire AMR through two fundamentally different genetic mechanisms. Spontaneous mutations that occur during the replication of the bacterial chromosome can make bacteria resistant to antibiotics, usually by modifying the cellular targets of antibiotics. Most pathogenic bacteria live in complex and densely populated

${ }^{1}$ Department of Zoology, University of Oxford, 11a Mansfield Rd., Oxford OX1 3SZ, UK. ${ }^{2}$ Department of Microbiology, Hospital Universitario Ramon y Cajal, IRYCIS and CIBERESP, Madrid, Spain. Email: craig.maclean@zoo.ox.ac.uk microbial communities (microbiomes) that are associated with human tissues, such as the gut, skin, and respiratory tract. The overwhelming majority of bacteria in these microbiomes are harmless commensals that do not cause disease. However, it has become clear that commensal bacteria provide a rich source of dedicated AMR genes (4). Mobile genetic elements, such as plasmids and bacteriophages (bacterial viruses), transfer these AMR genes between bacterial populations through the process of horizontal gene transfer (HGT) (see the figure). Crucially, HGT allows pathogenic bacteria to evolve resistance by acquiring pre-existing AMR genes from commensal bacteria. The basic mechanisms of horizontal gene transfer were discovered over 50 years ago, and the current challenges for this field are to better understand the rate at which pathogens acquire AMR genes by HGT, and to identify the key conduits that transfer AMR genes from commensal bacteria to pathogens. For example, recent studies have identified new pathways for HGT, such as co-lateral transduction, which drives the rapid transfer of chromosomal DNA by phage (5). It is possible that studying patterns of distribution of AMR genes in microbiomes combined with experiments that track the acquisition of AMR genes in complex communities, could help to start solving this puzzle.

One of the key insights from clinical microbial sequencing studies is that the rise of AMR in most important human pathogens has been largely driven by the spread of a small number of successful strains that can be effectively transmitted between patients. The acquisition of mobile elements that confer resistance to multiple antibiotics appears to be the key genetic mechanism that has enabled the dissemination of these globally prevalent AMR superbugs (2). For example, Staphylococcus aureus has evolved by acquiring staphylococcal cassette chromosome mec elements that confer resistance to a broad spectrum of antibiotics, most notably methicillin. Additionally, the dominant enteric pathogens Escherichia coli and Klebsiella pneumoniae have evolved resistance to the most recent generation of $\mathrm{B}$ lactam antibiotics by acquiring plasmids carrying extended-spectrum ß-lactamases and carbapenemases (6). Experimental studies have largely focused on studying the evolution of AMR by chromosomal mutations, often focusing on resistance mutations that have little, in any, clinical relevance. AMR is entirely caused by mutation in Mycobacterium tuberculosis, and mutations that confer resistance to fluoroquinolone antibiotics are important in the emergence of a broad spectrum of multi-drug resistant pathogens. However, it is important to note the discrepancy between these disciplines with clinical studies focused on mobile elements and experimental studies focusing on mutational AMR.

A key insight from experimental studies has been that the acquisition of AMR, either by mutation or by HGT, creates a fitness cost that is reflected in reduced growth rate and virulence $(7,8)$. This cost is thought to be the main obstacle that limits the spread of AMR by restricting the ability of resistant bacteria to successfully transmit to new hosts and establish infections. Pioneering experimental studies demonstrated that bacteria and/or mobile elements can evolve compensatory adaptations that eliminate the cost of resistance (7). Compensatory evolution allows AMR to stably persist in bacterial populations, even in the absence of antibiotic use, suggesting that compensation may be a key mechanism that drives the spread of successful AMR strains(9). A widely advocated solution to the AMR crisis is to restrict consumption, but the efficacy of this intervention is likely to be strongly dependent on the fitness of AMR bacteria. For example, reducing antibiotic use could have minimal effects on the prevalence of AMR if compensatory evolution has already occurred in dominant resistant strains.

Hundreds of studies have investigated the fitness of laboratory-evolved AMR, but understanding of the fitness of clinical pathogens remains limited (8). For example, few studies have measured the fitness of 'naturally' evolved AMR in clinically-isolated strains, and evidence of compensatory evolution has only been found in a few pathogens, such as Mycobacterium tuberculosis (10). This is particularly important for plasmid-mediated AMR: Even though plasmids are the most important mechanism of AMR 
acquisition in pathogens (2), the fitness effects of important AMR plasmids in their natural bacterial hosts is unknown. Clinical strains are difficult to manipulate genetically, making it difficult to measure the contribution of individual AMR genes to fitness using existing methods. New technological developments, such as CRISPR-based genomic engineering, allow gene editing and even completely removing AMR plasmids from clinical strains, potentially resolving this limitation (11). A further challenge will be to move beyond the broth culture systems that are currently used to measure fitness experimentally by developing model systems that capture key elements of the infection biology of bacterial pathogens. Ideally, these systems should include abiotic stressors that pathogens encounter during infections (resources, stressors, etc.). as well as interactions with commensal microbes that may promote or restrict the growth of AMR pathogens.

One widely advocated strategy to try to stop the spread of AMR is to use multidrug combination therapies to suppress or reverse selection for resistance (12). Recent work has focused on understanding how collateral sensitivity, which occurs when increased resistance to one antibiotic confers increased sensitivity to a second antibiotic, can be used to design antibiotic combinations that select against resistance. Important mobile AMR genes confer collateral sensitivities (13), and AMR plasmids produce common metabolic alterations in their bacterial hosts (14), suggesting that it might be possible to identify exploitable collateral sensitivity to clinically important mobile genetic elements.

An alternative approach to combat HGTacquired AR is to exploit naturally occurring barriers to the transfer of AMR genes. Bacteria possess an arsenal of xenogenic defence mechanisms, including both innate (restriction-modification) and adaptive (CRISPR-Cas) immune systems that recognise and destroy incoming 'non-self DNA (15). The native function of these systems is to protect bacteria from parasitic mobile elements, such as lytic bacteriophage, but they can be engineered to generate sequencespecific antimicrobials that target mobile AMR elements (11). In particular, CRISPRCas based systems have potential to genetically manipulate pathogen populations. A key challenge of using this approach is to develop tools to effectively deliver engineered CRISPR-Cas systems to pathogenic bacteria. AMR illustrates the incredible power of mobile genetic elements to introduce new genes into pathogen populations, and an improved understanding of HGT of resistance could ultimately help pave the way for the efficient delivery of 'anti-resistance' systems. Understanding the evolutionary drivers of AMR could make a substantial contribution to preserving the efficacy of next generation antimicrobials, but realizing this potential will require a fundamental shift in how evolutionary biologists think about, and study, AMR.

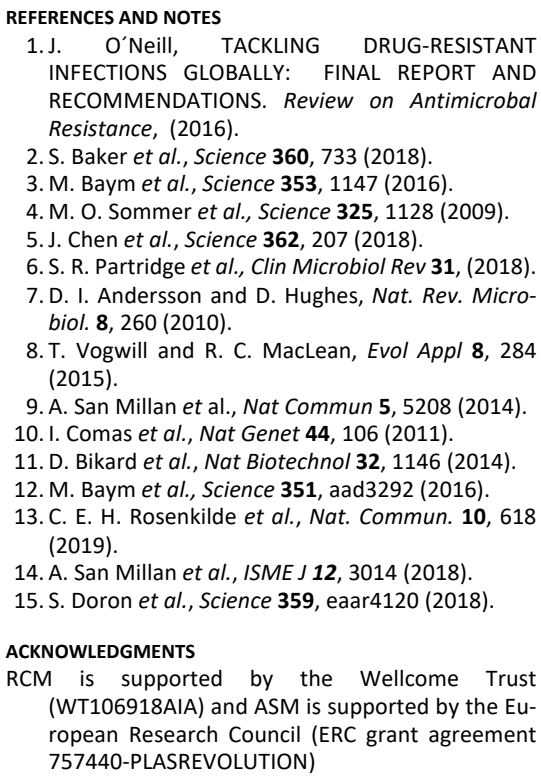

$10.1126 /$ science aax3879

Mechanisms of acquisition and stabilization of mobile antibiotic resistance

Pathogenic bacteria acquire antibiotic resistance (AMR) genes through two main mechanisms of horizontal gene transfer (HGT). Plasmids carrying AMR genes can be transferred into bacteria through conjugation. Or, bacteriophages can 'accidentally' package AMR genes from a cell that they have infected, and transfer these genes to closely related bacteria (transduction). Compensatory mutations alleviate the fitness costs imposed by mobile genetic elements, contributing to their stabilization. CRISPR-Cas based systems may selectively destroy DNA carrying mobile AMR genes. 